2002-2009, 0.71/10,000 in 2010-2013). A cluster of cases was identified in Lanarkshire where the incidence was $1.26 / 10,000$ live births, significantly higher than the rest of Scotland $(0.59$ / 10,000, $\mathrm{p}=0.033)$.

Conclusion BA atresia incidence appears relatively stable in Scotland but with an unexplained cluster of cases in Lanarkshire; examination of this cluster may provide epidemiological insight into disease pathogenesis Despite a significant reduction in time to Kasai, the 2YTFS in Scotland remains disappointing, is lower than the pre-rationalisation figure of $65 \%$ and is not currently an endorsement of centralisation of BA surgery in Scotland.

\section{G361 PARENTAL PHOTOGRAPHIC MONITORING OF ASCITES}

C MacDougall, L Saych, A Brightwell, K Blair, P Clarke, G Briars. Paediatrics, Norfolk and Norwich University Hospital, Norwich, UK

\subsection{6/archdischild-2015-308599.317}

Aims Primary Chylous Ascites is a rare cause of abdominal distension in infants. Our patient had six weeks of intravenous octreotide and total parenteral nutrition followed by medium chain triglyceride formula feeding. We describe his monitoring by girth measurement and parental photography.

Methods A four week old boy who presented with worsening abdominal distension and discomfort had ultrasound confirmed ascites. At paracentesis, $100 \mathrm{ml}$ of chylous fluid was aspirated. His clinical course was monitored by serial girth measurement and parental photography using a modern mobile telephone.

Results Variability in abdominal circumference measurements (Figure 1) made detection of clinical improvement more difficult than it was with serial photography (Figure 2). His mother found the photographs helpful in monitoring her son's progress. Conclusion This superiority of parental photography over formalised girth measurement monitoring in our patient mandates critical review of these techniques. Informal parental photography has been used in assessment of dermatological conditions ${ }^{1}$ and inguinal hernias. ${ }^{2}$ Inter-observer variability in measurements of abdominal circumference is recognised. ${ }^{3}$ Parental photography may increase parents' perceived involvement in their child's care in such chronic treatment and promote a family centred approach. We speculate that measurements from technical photographs ${ }^{4}$ as employed in sports biomechanical studies ${ }^{5}$ will be the most sensitive tool for clinical assessment of ascites.

\section{REFERENCES}

1 Nayler JR. Clinical photography: a guide for the clinician. I Postgrad Med. 2003:49:256-262

2 Kawaguchi AL. Inguinal hernias can be accurately diagnosed using the parent's digital photographs when the physical examination is nondiagnostic. J Pediatr surg. 2009;44:2327-2329

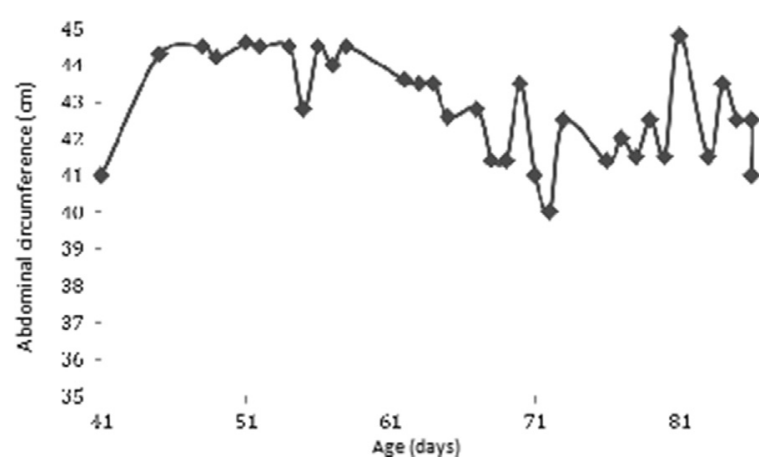

Abstract G361 Figure 1 Abdominal circumference measurements

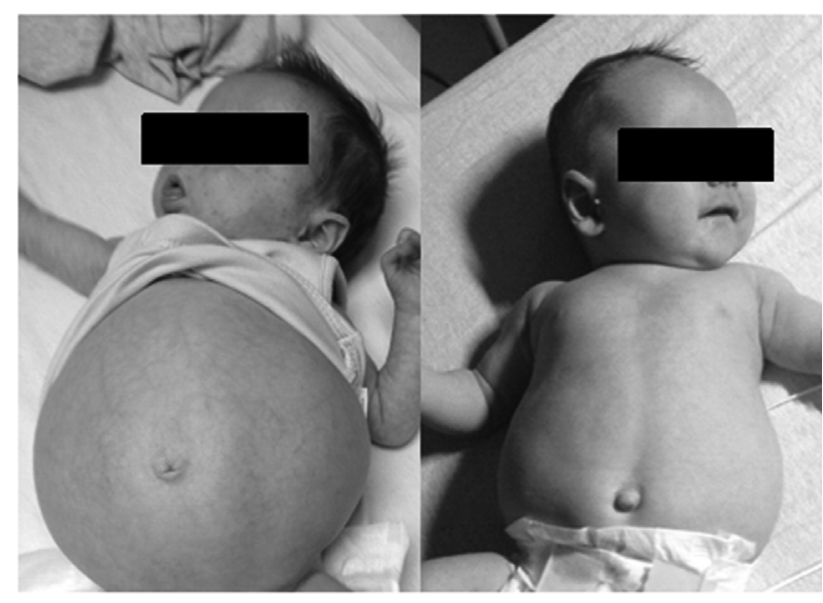

Abstract G361 Figure 2 Clinical improvement via photography

3 Johnson TS, Engstrom JL, Gelhar DK. Intra and interexaminer reliability of anthropometric measurements of term infants. I Pediatr Gastroenterol Nutr. 1997;24:497-505

4 Jensen RK. Estimation of the biomechanical properties of three body types using a photogrammetric method. J Biomech. 1978;11:349-358

5 Wicke J, Lopers B. Validation of the volume function with Jensen's (1978) elliptical cylinder model. J App/ Biomech. 2003;19:3-12

\section{G362(P) CHILD NUTRITION: A “NEED TO FEED” FUTURE DOCTORS}

${ }^{1} \mathrm{~S}$ Bali, ${ }^{2} \mathrm{H}$ Jacob, ${ }^{2} \mathrm{C}$ Fertleman. ${ }^{1}$ Paediatric Accident and Emergency, Chelsea and Westminster Hospital, London, UK; ${ }^{2}$ Department of Paediatrics, The Whittington Hospital, London, UK

\subsection{6/archdischild-2015-308599.318}

Aims Parents may ask doctors working in a range of specialties for advice on childhood nutrition. The Department of Health states that all doctors working with children should engage in health promotion. Ensuring the provision of high quality information may help prevent obesity and optimise the health of children and young people.

As many doctors may have limited postgraduate exposure to paediatrics, medical school is an ideal time to learn about the importance of childhood nutrition. We decided to explore the knowledge, skills and attitudes of medical students about providing guidance on childhood nutrition.

Methods We sent a survey to all medical students undertaking their paediatric placement at one teaching hospital between 2012-13. We asked the students whether they felt comfortable advising about breastfeeding, infant and toddler nutrition, obesity, food allergies and infant weighing schedules. We also enquired from them about maternal alcohol consumption during breastfeeding and about a crying baby whose mother was struggling to breastfeed.

We collected data using software from SurveyMonkey ${ }^{\mathrm{TM}}$ and analysed it using Microsoft Excel $^{\mathrm{TM}}$.

Results 82 students were asked to fill in the survey. 47(57\%) responded. 32(68\%) felt uncomfortable advising about breastfeeding and toddler nutrition. Despite this, 33(70\%) felt they would advise a mother struggling with breastfeeding rather than referring her to the health visitor. $32(68 \%)$ and $26(55 \%)$ thought that with help they could provide information about obesity and food allergies respectively. Regarding maternal alcohol consumption whilst breastfeeding 27(57\%) knew the appropriate advice about drinking minimally or in moderation. 